\title{
A Solar Coronal Jet Event Triggers A Coronal Mass Ejection
}

\author{
Jiajia Liu, Yuming Wang, Chenglong Shen, Kai Liu, Zonghao Pan, and S. Wang
}

CAS Key Laboratory of Geospace Environment, Earh and Space Science School, University of Science and Technology of China, NO. 96, JinZhai Road, Hefei, Anhui 230026, China

\section{Contents}

\section{Introduction}

2 Observations

3 Mechanisms and Discussions

4 Conclusions

\section{Abstract}

We present the multi-point and multi-wavelength observation and analysis on a solar coronal jet and coronal mass ejection (CME) event in this paper. Employing the GCS model, we obtained the real (three-dimensional) heliocentric distance and direction of the CME and found it propagate in a high speed over $1000 \mathrm{~km} \mathrm{~s}^{-1}$. The jet erupted before and shared the same source region with the CME. The temporal and spacial relationship between them guide us the possibility that the jet triggered the CME and became its core. This scenario could promisingly enrich our understanding on the triggering mechanism of coronal mass ejections and their relations with coronal large-scale jets. On the other hand, the magnetic field configuration of the source region observed by the SDO/HMI instrument and the offlimb inverse Y-shaped configuration observed by SDO/AIA 171 $\AA$ passband, together provide the first detailed observation on the three-dimensional reconnection process of large-scale jets as simulated in Pariat et al. 2009. The erupting process of the jet highlights that filament-like materials are important during the eruption not only of small-scale X-ray jets (Sterling et al. 2015) but also probably of large-scale EUV jets. Based on our observation and analysis, we propose a most possible mechanism for the whole event with a blob structure overlaying the three-dimensional structure of the jet to describe the interaction between the jet and the CME.

\section{Introduction}

As one of the most intriguing phenomena acting in the solar atmosphere, solar jets have been studied extensively and deeply in the past few decades [e.g., Shibata et al., 1996; Moore et al., 2010; Cirtain et al., 2007; De Pontieu et al., 2007]. Despite the different properties in dominant temperature, scale and dynamics, they are thought to be import in releasing solar magnetic free energy through reconnection [e.g., Shibata et al., 2007; Pariat et al., 2009; Liu et al., 2014; Fang et al., 2014] and contributing in corona heating and/or solar wind acceleration [e.g., Shibata et al., 2007; Liu et al., 2014; McIntosh et al., 2010; Tsiropoula and Tziotziou, 2004;
Liu et al., 2015]. As done by the numerical simulations in Pariat et al. [2009], the triggering mechanism of solar jets could be credited to reconnection occurring within an inverse Y-shaped threedimensional magnetic field configuration. However, direct observations on the detailed evolution of such a $3 \mathrm{D}$ reconnection are still absent.

Playing impressive roles in affecting the Earth environment, 1 coronal mass ejections (CMEs) have attracted great attentions since the era of space physics [Low, 2001, as a review]. As a signifi-

1 cant numerous matter ejected from the Sun, CMEs have been studied thoroughly in such as their observational features [e.g., Wood

2 et al., 1999], models [e.g., Priest and Forbes, 1990; Lin and Forbes, 2000], early evolution [e.g., Liu et al., 2014], interactions with each

6 other [e.g., Shen et al., 2012] and their arrival at the Earth [e.g., Shen et al., 2014]. As widely believed in the community, a substantial part of CMEs are found to be associated with prominence/filament eruptions [e.g., Gopalswamy et al., 2003; Gilbert et al., 2000].

What hides behind the different typical geometries of jets (elongated) and CMEs (blob-like) is the distinct magnetic field configurations. While jets are usually believed to erupted along open field lines [e.g., Shibata et al., 1996], CMEs are thought to be associated with closed helical fields [e.g., Chen et al., 1997]. Potential relations and interactions between these two glamorous phenomena (jets and CMEs) then will be intriguing and enrich our knowledge on the various physical processes in the solar atmosphere. Previous studies have shown that some (narrow) CMEs could be the extension of large-scale solar jets in white-light coronagraphs [e.g., Wang et al., 1998; Liu, 2008]. Other possibilities might be one of them is triggered by the other. However, do these possibilities exist veritably in the solar atmosphere? How will such an interaction between a CME and a jet happen? Corresponding observations and analysis have not yet been performed.

In this paper, we will present the multi-point and multiwavelength observation and analysis on a jet and CME event. The jet was originated from a single positive polarity active region, driven by a 3D magnetic topology which surprisingly resembled that in Pariat et al. [2009], providing the first detailed observation on the evolution of such a 3D-reconnection triggering mechanism for solar jets. The CME is found to be triggered by the jet event with the jet becoming its core, illustrating a scenario in which these two eruptive events could be closely related. Conclusions come in Sect. 4 based on the observations in Sect. 2 and discussions in Sect. 3.

\section{Observations}

During the (quasi-) frontal travel of the active region NOAA 11644 (Fig. 1 (C)), it performed several attractive eruptions, among which the most intriguing one is what acted after 19:00 UT on the 
day January $15^{\text {th }}$ 2013. A fast Coronal Mass Ejection (CME) with speed over 1000 kilometers per second could be observed simultaneously by the coronagraphs LASCO C2/C3 onboard the SOlar Heliospheric Observatory (SOHO) and COR1/COR2 onboard both probes of the Solar TErrestrial RElations Observatory [STEREO, Kaiser et al., 2008] (Fig. 1, online movie M1). Just before the CME, a solar coronal EUV jet was observed in the field-of-view (FOV) of the AIA instrument onboard the Solar Dynamics Observatory [SDO, Pesnell et al., 2012] and EUVI instruments onboard both of the STEREO probes (Fig. 1, online movie M2). Figure 1 (B) and (C), together with the online movie M2, show the great simultaneity between the observed jet via SDO AIA and the eruption activities within the active region NOAA 11644 via STEREO-A EUVI, indicating that the active region should be the source region of the jet. Location of the center of the active region was about $\mathrm{E} 10^{\circ} \mathrm{N} 24^{\circ}$ in the FOV of the STEREO-A EUVI instrument. As STEREO-A was about $129^{\circ}$ ahead the earth (and SDO and SOHO) at 19:00 UT, the longitude of the center of the active region seen from SDO should be W119 ${ }^{\circ}$.

The CME appeared at the north-east limb around 19:40 UT in the FOV of the STEREO-B (STB) COR1 (Fig. 1 (A)), which was about $134^{\circ}$ behind the earth (and SOHO and SDO). Coronagraph LASCO C2 captured the first image of the CME at around 20:00 UT and half an hour later LASCO C3 and STEREO COR2. To obtain the three-dimensional (real) direction, position and velocity of the CME, we employ a forward reconstruction model (the GCS model) proposed by Thernisien et al. [2009], which assumes a self-similar expanding flux rope structure of the target CME. We then compare the resulting flux rope obtained from the GCS model with the images from LASCO C2/C3 and STA/STB COR2 at six instances, when we could at least partially identify the leading edge of the CME in all the three images (LASCO C2, STA COR2 and STB COR2), to make the best agreement between the flux rope and the CME. Parameters of the flux rope could tell us the real heights (heliocentric distances), longitudes and latitudes of the CME at different times. One of them at 20:30 UT is shown in panel (G)-(I) in Figure 1, with the green dotted wires the surface of the flux rope structure.

The results display that the latitudes and longitudes of the CME stayed almost invariable around $\mathrm{N} 48^{\circ}$ and $\mathrm{W} 120^{\circ}$ seen from the Earth (and SDO) throughout its travel from 6 Rs to 20 Rs. A parabolic fitting between the heliocentric distance of the CME's leading edge and the corresponding time shows an average propagating speed of the CME around $1031 \mathrm{~km} \mathrm{~s}^{-1}$.

On the other hand, continuous small brightenings could be figured out since 19:16 UT in the AIA $304 \AA$ passband images around $\mathrm{N} 24^{\circ}$ at the north-west limb and the first sign of plasma eruption which formed a jet appeared around 19:32 UT (Fig. 1 (B), online movie M2 \& M3), few minutes earlier than when the CME appeared in the FOV at the STEREO COR1 observations (19:40 UT). Most of the jet materials were seen in the AIA $304 \AA$ passband and rarely found in passbands with characteristic temperature above 2 MK [211 ̊, Lemen et al., 2012], indicating that the jet was majorly formed with chromospheric cool materials (online move M2). The jet kept rising along a trajectory which was about $28^{\circ}$ counterclockwise away from the local radial direction and didn't show any signal of falling back. The position angle of the jet was almost the same with that of the CME (online movie M1)

The similarity of the position and the temporal relationship between the jet and the CME, suggest that there should be some close relations between them: (1) the CME was the outer manifestation of the jet in the coronagraphs, (2) the CME was a bulk of materials that was triggered by the jet event, and (3) the lifting of the CME blob triggered footpoint region activities and the eruption of the jet. The third one could be directly excluded because the activities which triggered the jet were more than 20 min earlier than when the CME was observed in the FOV of STB COR1 (19:16 UT vs. 19:40 UT). To figure out which situation among the first two the truth is, we placed a slit along the CME's direction in the STB COR1 images (Fig. 1 (D)), a slit along the jet's axis in the AIA images (Fig. 1 (E)) and a slit along the CME's direction in the LASCO C2/C3 images (Fig. $1(\mathrm{H})$ ) which was the extension of the slit in the AIA images. The corresponding time-distance plots are shown in Figure 2 with the projection effect corrected based on the GCS result.

Obviously, the CME could be found in all the three runningdifference time-distance diagrams for STB COR1 (Fig. 2 (C)) and LASCO C2/C3 (Fig. 2 (B) and (A)) and they show very high consistency. Fitting the leading edge of the CME in the time-distance plots of these coronagraphs via a linear function yields the average velocity of it about $1064 \pm 33 \mathrm{~km} \mathrm{~s}^{-1}$, which is highly consistent with the GCS result $\left(1031 \mathrm{~km} \mathrm{~s}^{-1}\right)$. Figure 2 (D) shows the corresponding running-difference time-distance plot of the AIA $304 \AA$ images with a deduced cadence of 10 min to make it comparable to the STB COR1 plot. It is shown that there was no clear manifestation of the CME in the AIA $304 \AA$ observations (nor in the other EUV passbands of AIA which is not shown here, see movie M2). And the time sequence shows that the CME was not the extension of the jet in the coronagraphs.

High-cadence (12 s) AIA images could hint us some detailed kinetics of the jet. Figure 2 (E) shows the de-projected runningdifference time-distance plot along the slit in Figure 1 (E) using the 12 s-cadence images of the AIA $304 \AA$ passband. Sub-jets were expelled successively, indicating that continuous reconnection happened around the source region of the jet as described in Moore et al. [2010] and observed in Liu et al. [2014]. The average axial speed of these sub-jets was about $189 \pm 42 \mathrm{~km} \mathrm{~s}^{-1}$ at the bottom (green dashed line in Fig. 2 (E)) and they could be found to undergo obvious acceleration. However, it is difficult for us to trace the track of a particular sub-jet in the time-distance plot to estimate the exact acceleration, due to the 20-min data gap from about 20:01 UT. The speed of the jet in the FOV of STB COR1 was found to be almost the same with that of the CME's leading edge, together with the great continuity between the AIA plot and coronagraph plots, indicates the second situation that the CME was triggered by the jet event with the jet becoming its core should reveal the truth.

\section{Mechanisms and Discussions}

The source region of the jet which was identified as the active region NOAA 11644 could be seen clearly from the STEREO-A EUVI $195 \AA$ A observations. Figure 3 (A)-(D) exhibit four different times before, during and after the eruption. Figure 3 (A) shows the active region before the eruption at 19:00 UT, with the red-blue contours the line-of-sight (LOS) photospheric magnetic fields observed by the HMI instrument onboard SDO at 17:00 UT nine days earlier when the active region was almost facing the satellite. It is shown clearly that the active region contained a single positive polarity surrounded by negative polarities, with another positive polarity at the lower left in a southern active region. Filament-like materials marked as a purple dashed curve could be found laying around the polarity inversion region between the single positive polarity and the surrounding negatives. Brightenings began around 19:15 UT as shown in panel (B) and developed into the eruption of the jet which was seemingly formed by the laying filament-like materials. The 

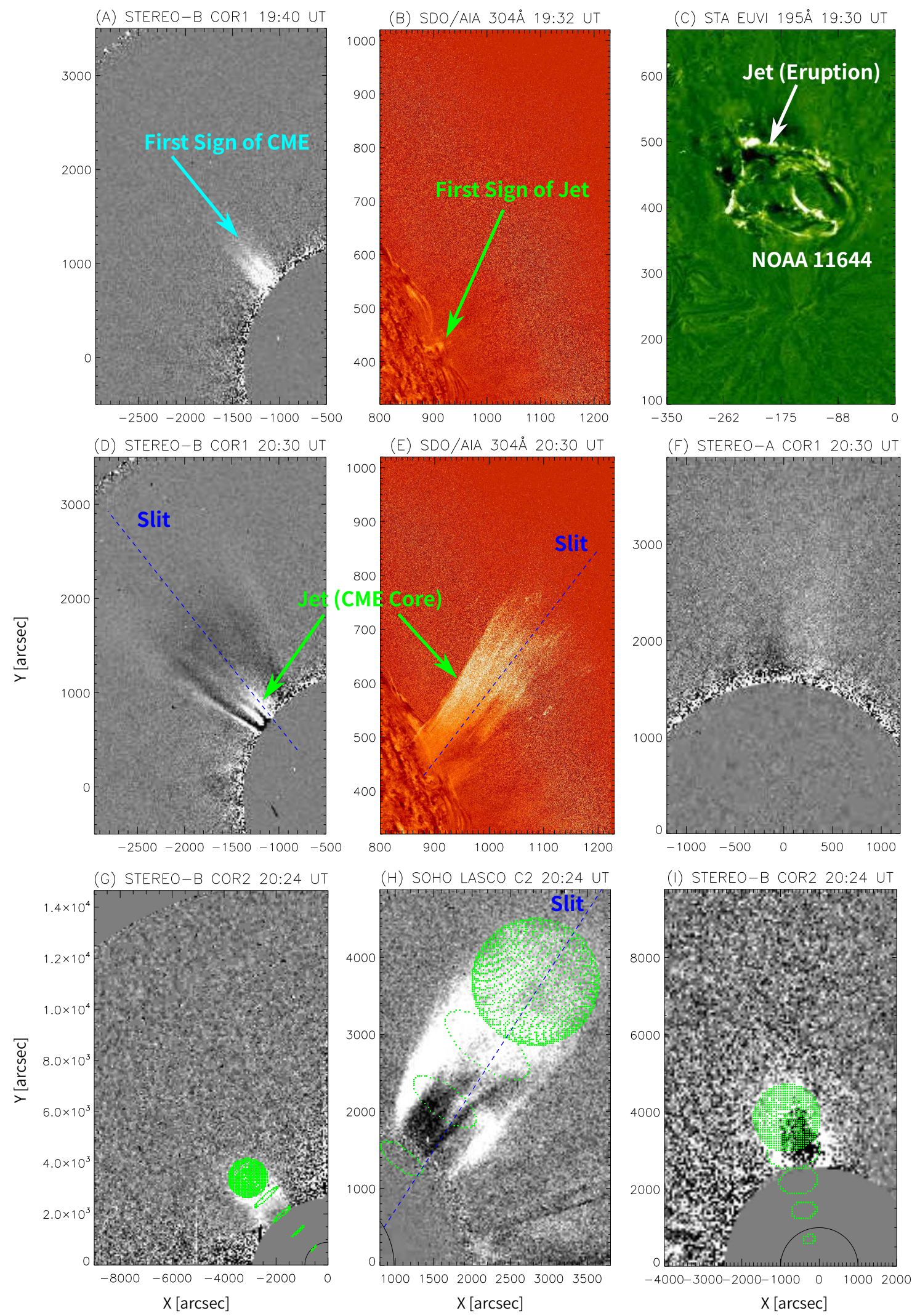

Figure 1: Multi-point observations on the jet and the CME by SDO AIA, SOHO LASCO and STEREO EUVI/COR1/COR2 instruments. The top three panels are the STEREO-B COR1 observation on the first sign of the CME at 19:40 UT, the SDO AIA $304 \AA$ observation on the first sign of the jet at 19:32 UT and the STEREO-A EUVI $195 \AA$ observation on the source region of the jet (active region NOAA 11644) at 19:30 UT, respectively. The medium three panels are the STEREO-A/B COR1 and SDO AIA $304 \AA$ passband observations at the same time 20:30 UT. The slits are selected along the axial direction of the jet or the CME. The bottom three panels are the simultaneous observations of the STEREO-A/B COR2 and SOHO LASCO C2 instruments on the CME on 20:24 UT. The green dotted wires are the resulting surface of the reconstured structure of the CME employing the GCS forward modeling method [see descriptions about the model in the text and more in Thernisien et al. 2009] 


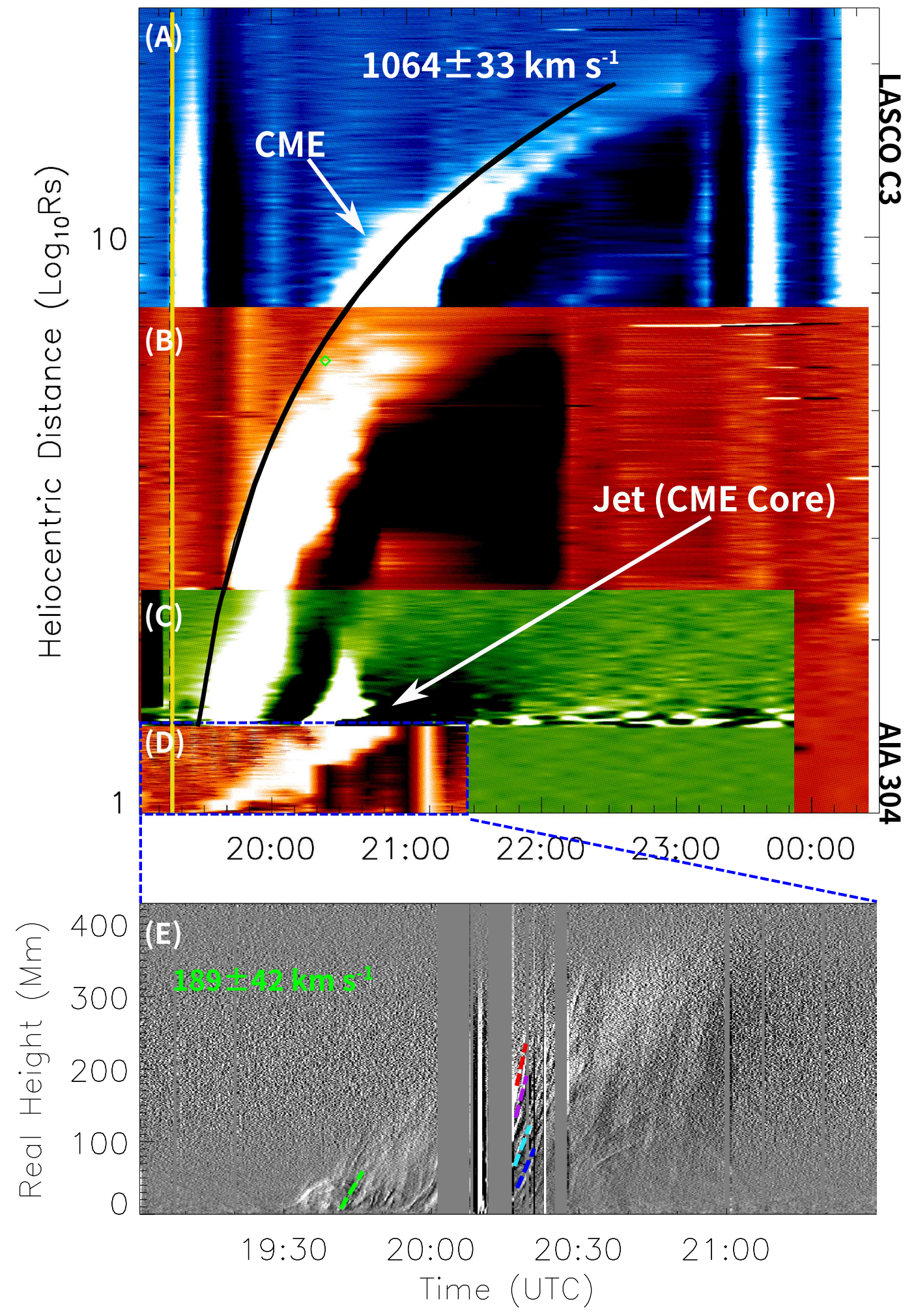

Figure 2: Panel (A)-(C): Running-difference time-distance plots of the slits shown in Fig. 1 based on data from SOHO LASCO C3, C2 and STEREO-B COR1 respectively. Panel (D)-(E): Running-difference time-distance plots of the cadence-reduced and original SDO AIA $304 \AA$ A passband data, respectively. The yellow vertical line across panel (A) to (D) indicates the time when the first brightening occurred at 19:16 UT inside the source region of the jet. The black solid curve across panel (A), (B) and (C) shows the fitted leading edge of the CME with a linear fit which results in an average speed of $1064 \mathrm{~km} \mathrm{~s}^{-1}$. The green diamod shown in panel (B) is the leading edge reconstructed via the GCS model at 20:24 UT in panel (D)-(F) in Fig. 1. Color-coded lines in panel (E) give examples of tracks and velocities of different sub-jets. 

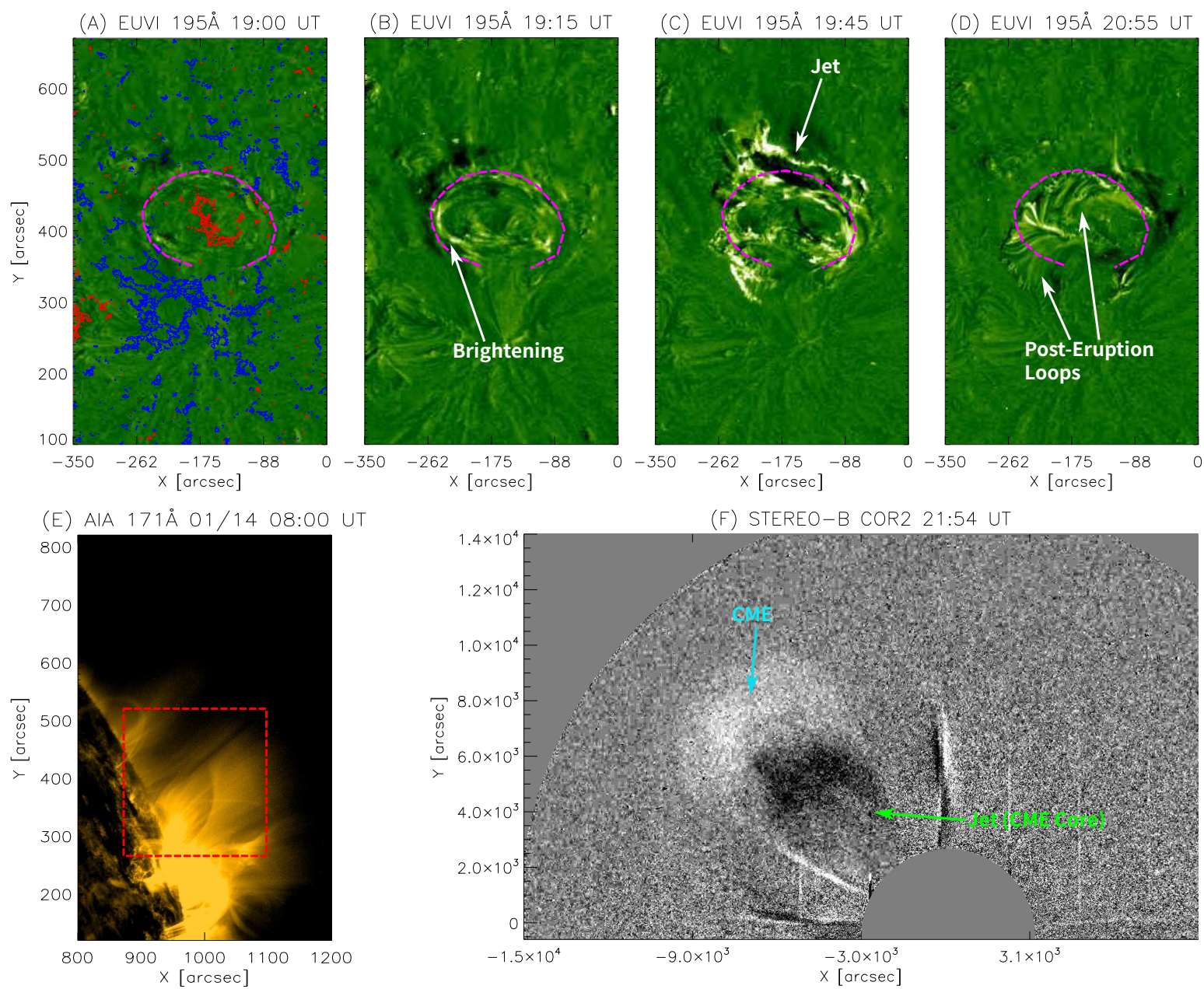

Figure 3: Panel (A)-(D): Time-sequence evolution of the source region (NOAA 11644) of the jet and the CME in the FOV of STEREO-A EUVI $195 \AA$ passband. The red and blue contours in (A) show the positive and negative magnetic fields observed by SDO HMI instrument nine days before the jet and CME event. The purple dashed curve marks the filament-like materials laying around the polarity inversion region before the eruption. Panel (E) is the SDO AIA 171 A image at 08:00 UT one day before the event, resolving an inverse Y-shaped configuration of the magnetic field lines enclosed in the red dashed rectangle. Panel (F): STEREO-B COR2 observation on the CME and the jet (CME core) at 21:54 UT.

eruption began around the left end of the purple curve, gradually proceeded clockwise and rooted around the right end, as shown in panel (C). Newly formed loops could also be notably observed after the eruption of the jet, exhibited in panel (D). As we can find from Movie 2 and Figure 3, the filament-like materials participated a lot during the triggering process and finally formed the erupted jet.

Figure 3 (E) shows the AIA $171 \AA$ observation at 08:00 UT one day before the event and not far after the active region turned to the limb. Images in the $171 \AA$ passband can mostly resolve the magnetic field topology in the lower corona and showed a clear inverse Y-shaped configuration (enclosed in the red dashed rectangle in Fig. 3 (E)), which almost kept exact pace with the simulations of solar jets in Pariat et al. [2009] and seemed like a magnified "anemone" jet's root observed in Shibata et al. [2007]. The photospheric magnetic field, the off-limb inverse Y-shaped configuration, the erupting process of the jet's materials and the existence of newly formed loops after the eruption are greatly analogous to the 3D-reconnection simulations in Pariat et al. [2009], inspiring us to surmise similar three-dimensional reconnections happening in this particular event.

Based on the above observation and analysis, we are then able to propose the most possible mechanism for the whole event, which is shown as a 2D-version sketch in Figure 4. Periphery field lines that originate from the single positive polarity inside the active region 11644 and end at the negative polarities form the inner fan (green fields in Fig. 4). Out of which are the bottom fields of the CME (cyan fields) originating from the southern positive polarity. If activities underneath the inner fields introduce any twist/shearing (shown as the blue field), magnetic free energy will be built up and reconnection will occur when the balance is broken up [Pariat et al., 2009; Fang et al., 2014]. Plasma materials speed up by the reconnection will then form a jet (gray-colored arrow). The bursts could also give a push to the blob structure upon the black dashed horizontal line. Due to the lack of observations on the early stage of the blob structure, we cannot know how the bursts pushed it in this particular event. Several effects such as: (1) the elevated inner fan due to the reconnections [Pariat et al., 2009] or/and (2) the accelerated jet materials could lead to the ascending of the blob. Under 


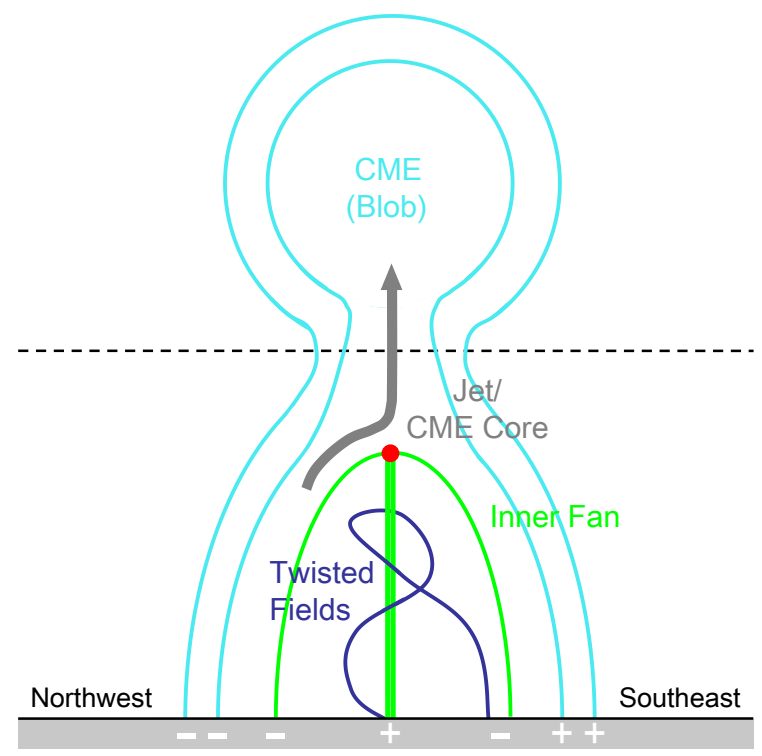

Figure 4: 2D-sketch vesion of the magnetic field configuration that results in the observed event and phenomena. See the text for details of the illustration on the mechanisms.

the effect of one or more of the above processes, the blob rises and forms the observed CME.

Like the the one modeled in Fang et al. [2014] and the one observed in Liu et al. [2014], rotational motions of the jet's material could also be observed from the AIA $304 \AA$ images in this event. Placing slits perpendicular to the jet's axis (which are not shown in the figures) allow us to estimate the rotating periods of the materials employing a sine-function fit as done in Liu et al. [2014]. Periods turn out to be about $15 \mathrm{~min}$ at the bottom with the resulting linear speed of about $280 \mathrm{~km} \mathrm{~s}^{-1}$. The periods became longer at the top of the jet in the FOV of AIA, indicating a deceleration of the rotational motion. As illustrated in Liu et al. [2014], the rotational motion comes with the release of residual magnetic free energy after the reconnection, how much the residual is would affect the rotational motion of the jet. Thus it is possible that in this particular case, the reconnection may have already released most of the magnetic free energy and few of them is left to drive the rotational motion.

However, another situation that the angular momentum of the jet was passed into that of the CME is not prohibited. As lack of direct observations on the rotational motion of the CME [discussions on this particular issue could be found in Tian et al., 2013, as an example], we could try to figure out if this situation is possible from the poloidal motion of magnetic clouds (MCs) which are the counterpart of CMEs in the interplanetary space. As derived from a velocity-modified cylindrical force-free flux rope model base on Wind observations, Wang et al. [2015] found that almost all the MCs more or less had shown poloidal motion with meridian linear speed around $10 \mathrm{~km} \mathrm{~s}^{-1}$. Assuming all the angular momentum of the jet has been passed to the CME, a self-expanding propagation of the CME and the conservation of angular momentum in the interplanetary space, the poloidal speed of the MC, evolved from the CME in this particular case, would be about $1.3 \mathrm{~km} \mathrm{~s}^{-1}$, which is slightly below but still not prohibited by the observations in Wang et al. [2015].

\section{Conclusions}

In this paper, we present the analysis of a coronal jet and a related coronal mass ejection (CME), employing multi-wavelength and multi-point observations from SDO AIA/HMI, STEREO EUVI/COR1/COR2 and SOHO LASCO C2/C3 instruments.

Detailed analysis has shown that there is a close relationship between the jet and the CME. Employing the GCS reconstruction model, we are able to obtain the real heights and positions of the $\mathrm{CME}$ at different times. It is found that the CME propagated with a speed over $1000 \mathrm{~km} \mathrm{~s}^{-1}$. After correcting the projection effect using results from the GCS model, we plot the time-distance diagrams of the CME in the FOV of STB/COR1 and LASCO C2/C3, and of the jet in the FOV of SDO/AIA. It is shown that the CME was not the extension of the jet in coronagraphs, but triggered by the jet event, with the jet becoming its core. All the observations indicate that a (high-speed) CME could also be triggered by an underneath jet event, which is quite different with classical models [e.g., Lin and Forbes, 2000] and provide a new viewpoint on studying the relations between these two different mass release events in the solar atmosphere.

The jet originated from a source region with single positive polarity surrounded by negative polarities. All the observational features and its source region configuration showed highly consistence with the three-dimensional reconnection simulation of solar jets in Pariat et al. [2009], providing the first detailed observations on such threedimensional reconnection triggering process of large-scale EUV jets. The erupting process of the jet observed by the STEREO-A $194 \AA$ instrument highlights the importance of the footpoint region filament-like materials in participating the eruption, which might provide another evidence on the model proposed by Sterling et al. [2015] (in which the authors showed evidences for X-ray smallscale jets).

Rotational motion of the jet materials could also be observed in the AIA $304 \AA$ images. The rotational motion was found to decelerate. The deceleration could be either caused by that: (1) little magnetic free energy was left after the reconnection, or/and (2) the angular momentum of the jet had been passed to the CME. As lack of observations on the temporal magnetic fields, early stages of the CME and in-situ data, we cannot exclude either one of these two explanations. On the other hand, as the CME was invisible in the observations of the AIA instrument due to the different temperature or/and the submarginal height, we are not able to figure out how exactly the CME interacted with the underneath jet event. This made the physical image incomplete in describing the interactions between the CME and the jet event of our picture in Figure 4. Future works on more observations and numerical simulations may shed light on the issues raised above.

Acknowledgments. We acknowledge the use of data from the Solar Dynamics Observatory (SDO), the Solar TErrestrial RElations Observatory (STEREO) and the SOlar Heliospheric Observatory (SOHO). Online movie M1 is generated using the "JHelioviewer" tool (http://www.helioviewer.org). This work is supported by grants from NSFC (41131065, 41121003 and 41574165), CAS (Key Research Program KZZD-EW-01-4), MOST 973 key project (2011CB811403), MOEC (20113402110001) and the fundamental research funds for the central universities. The research leading to these results has also received funding from NSFC 41274173, 41404134 and 41304145.

\section{References}

Chen, J., R. A. Howard, G. E. Brueckner, R. Santoro, J. Krall, S. E. Paswaters, O. C. St. Cyr, R. Schwenn, P. Lamy, and G. M. Simnett, Evidence 
of an Erupting Magnetic Flux Rope: LASCO Coronal Mass Ejection of 1997 April 13, ApJ Letters, 490, L191-L194, 1997.

Cirtain, J. W., L. Golub, L. Lundquist, A. van Ballegooijen, A. Savcheva, M. Shimojo, E. DeLuca, S. Tsuneta, T. Sakao, K. Reeves, M. Weber, R. Kano, N. Narukage, and K. Shibasaki, Evidence for Alfvén Waves in Solar X-ray Jets, Science, 318, 1580-, doi:10.1126/science.1147050, 2007.

De Pontieu, B., S. McIntosh, V. H. Hansteen, M. Carlsson, C. J. Schrijver, T. D. Tarbell, A. M. Title, R. A. Shine, Y. Suematsu, S. Tsuneta, Y. Katsukawa, K. Ichimoto, T. Shimizu, and S. Nagata, A Tale of Two Spicules: The Impact of Spicules on the Magnetic Chromosphere, PASJ, 59, 655, 2007.

Fang, F., Y. Fan, and S. W. McIntosh, Rotating Solar Jets in Simulations of Flux Emergence with Thermal Conduction, ApJ Letters, 789, L19, doi: $10.1088 / 2041-8205 / 789 / 1 / \mathrm{L} 19,2014$

Gilbert, H. R., T. E. Holzer, J. T. Burkepile, and A. J. Hundhausen, Active and Eruptive Prominences and Their Relationship to Coronal Mass Ejections, ApJ, 537, 503-515, doi:10.1086/309030, 2000.

Gopalswamy, N., M. Shimojo, W. Lu, S. Yashiro, K. Shibasaki, and R. A. Howard, Prominence Eruptions and Coronal Mass Ejection: A Statistical Study Using Microwave Observations, ApJ, 586, 562-578, doi:10.1086/ 367614, 2003

Kaiser, M. L., T. A. Kucera, J. M. Davila, O. C. St. Cyr, M. Guhathakurta, and E. Christian, The STEREO Mission: An Introduction, SSR, 136, 516, doi:10.1007/s11214-007-9277-0, 2008.

Lemen, J. R., A. M. Title, D. J. Akin, P. F. Boerner, C. Chou, J. F. Drake, D. W. Duncan, C. G. Edwards, F. M. Friedlaender, G. F. Heyman, N. E. Hurlburt, N. L. Katz, G. D. Kushner, M. Levay, R. W. Lindgren, D. P. Mathur, E. L. McFeaters, S. Mitchell, R. A. Rehse, C. J. Schrijver, L. A. Springer, R. A. Stern, T. D. Tarbell, J.-P. Wuelser, C. J. Wolfson, C. Yanari, J. A. Bookbinder, P. N. Cheimets, D. Caldwell, E. E. Deluca, R. Gates, L. Golub, S. Park, W. A. Podgorski, R. I. Bush, P. H. Scherrer, M. A. Gummin, P. Smith, G. Auker, P. Jerram, P. Pool, R. Soufli, D. L. Windt, S. Beardsley, M. Clapp, J. Lang, and N. Waltham, The Atmospheric Imaging Assembly (AIA) on the Solar Dynamics Observatory (SDO), Solar Phys., 275, 17-40, 2012.

Lin, J., and T. G. Forbes, Effects of reconnection on the coronal mass ejection process, JGR, 105, 2375-2392, doi:10.1029/1999JA900477, 2000.

Liu, J., Y. Wang, R. Liu, Q. Zhang, K. Liu, C. Shen, and S. Wang, When and how does a Prominence-like Jet Gain Kinetic Energy?, ApJ, 782, 94, 2014.

Liu, J., F. Fang, Y. Wang, S. W. McIntosh, Y. Fan, and Q. Zhang, On the Observation and Simulation of Solar Coronal Twin Jets, ApJ, Under Review, 2015.

Liu, R., Y. Wang, and C. Shen, Early evolution of an energetic coronal mass ejection and its relation to euv waves, The Astrophysical Journal, 797(1), 37, 2014.

Liu, Y., A Study of Surges: II. On the Relationship between Chromospheric Surges and Coronal Mass Ejections, Solar Phys., 249, 75-84, doi:10. 1007/s11207-008-9176-x, 2008.

Low, B. C., Coronal mass ejections, magnetic flux ropes, and solar magnetism, Journal of Geophysical Research: Space Physics, 106(A11), 25,141-25,163, doi:10.1029/2000JA004015, 2001.

McIntosh, S. W., D. E. Innes, B. de Pontieu, and R. J. Leamon, STEREO observations of quasi-periodically driven high velocity outflows in polar plumes, AAP, 510, L2, doi:10.1051/0004-6361/200913699, 2010.

Moore, R. L., J. W. Cirtain, A. C. Sterling, and D. A. Falconer, Dichotomy of Solar Coronal Jets: Standard Jets and Blowout Jets, ApJ, 720, 757770, doi:10.1088/0004-637X/720/1/757, 2010.

Pariat, E., S. K. Antiochos, and C. R. DeVore, A Model for Solar Polar Jets, ApJ, 691, 61-74, doi:10.1088/0004-637X/691/1/61, 2009.
Pesnell, W. D., B. J. Thompson, and P. C. Chamberlin, The Solar Dynamics Observatory (SDO), Solar Phys., 275, 3-15, doi:10.1007/ s11207-011-9841-3, 2012.

Priest, E. R., and T. G. Forbes, Magnetic field evolution during prominence eruptions and two-ribbon flares, Solar Phys., 126, 319-350, doi:10.1007/ BF00153054, 1990

Shen, C., Y. Wang, S. Wang, Y. Liu, R. Liu, A. Vourlidas, B. Miao, P. Ye, J. Liu, and Z. Zhou, Super-elastic collision of large-scale magnetized plasmoids in the heliosphere, Nature Physics, 8(12), 923-928, 2012.

Shen, C., Y. Wang, Z. Pan, B. Miao, P. Ye, and S. Wang, Full-halo coronal mass ejections: Arrival at the Earth, Journal of Geophysical Research (Space Physics), 119, 5107-5116, doi:10.1002/2014JA020001, 2014.

Shibata, K., M. Shimojo, T. Yokoyama, and M. Ohyama, Theory and Observations of X-Ray Jets., in Astronomical Society of the Pacific Conference Series, Astronomical Society of the Pacific Conference Series, vol. 111, pp. 29-38, 1996.

Shibata, K., T. Nakamura, T. Matsumoto, K. Otsuji, T. J. Okamoto, N. Nishizuka, T. Kawate, H. Watanabe, S. Nagata, S. UeNo, R. Kitai, S. Nozawa, S. Tsuneta, Y. Suematsu, K. Ichimoto, T. Shimizu, Y. Katsukawa, T. D. Tarbell, T. E. Berger, B. W. Lites, R. A. Shine, and A. M. Title, Chromospheric Anemone Jets as Evidence of Ubiquitous Reconnection, Science, 318, 1591-, doi:10.1126/science.1146708, 2007.

Sterling, A. C., R. L. Moore, D. A. Falconer, and M. Adams, Small-scale filament eruptions as the driver of X-ray jets in solar coronal holes, Nature, $523,437-440,2015$.

Thernisien, A., A. Vourlidas, and R. A. Howard, Forward Modeling of Coronal Mass Ejections Using STEREO/SECCHI Data, Solar Phys., 256, 111-130, doi:10.1007/s11207-009-9346-5, 2009.

Tian, H., S. Tomczyk, S. W. McIntosh, C. Bethge, G. de Toma, and S. Gibson, Observations of Coronal Mass Ejections with the Coronal Multichannel Polarimeter, Solar Phys., 288, 637-650, doi:10.1007/ s11207-013-0317-5, 2013.

Tsiropoula, G., and K. Tziotziou, The role of chromospheric mottles in the mass balance and heating of the solar atmosphere, AAP, 424, 279-288, doi:10.1051/0004-6361:20035794, 2004.

Wang, Y., Z. Zhou, C. Shen, R. Liu, and S. Wang, Investigating plasma motion of magnetic clouds at $1 \mathrm{AU}$ through a velocity-modified cylindrical force-free flux rope model, JGR, 120, 1543-1565, 2015.

Wang, Y.-M., N. R. Sheeley, Jr., D. G. Socker, R. A. Howard, G. E. Brueckner, D. J. Michels, D. Moses, O. C. St. Cyr, A. Llebaria, and J.-P. Delaboudinière, Observations of Correlated White-Light and ExtremeUltraviolet Jets from Polar Coronal Holes, ApJ, 508, 899-907, doi: 10.1086/306450, 1998.

Wood, B. E., M. Karovska, J. Chen, G. E. Brueckner, J. W. Cook, and R. A. Howard, Comparison of Two Coronal Mass Ejections Observed by EIT and LASCO with a Model of an Erupting Magnetic Flux Rope, ApJ, 512, 484-495, doi:10.1086/306758, 1999. 